Mr. Gale's announcement had been published, and, although undoubtedly authentic, were not of a character which would affect the credit justly due to Mr. Gale for the finding of that comet.

It is to be hoped that the comet which Mr. Lowe has Adelaide Observatory, South Australia, I9 I3 Jan. 23. reported may be picked up again when it is in a more favourable position, or may perhaps be identified on photographs which are already in existence. It is therefore suggested that if there are any photographs of the region in question near the given dates they may repay examination.

\title{
Mitteilungen über die Nova Geminorum 2.
}

Die Beobachtungen der Nova sind angestellt mit dem bloßen Auge, einem Trieder-Binokel (Silvamar) von Zeiß und dem Sucher des Zehnzöllers. Die Helligkeit der Nova ist zwischen die der Vergleichsterne interpoliert, aber mit den Interpolationszahlen ist zu gleicher Zeit ein Stufenwert verbunden.

Die Vergleichsterne sind:

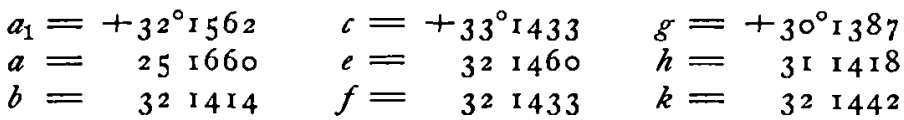

Die Helligkeit der Vergleichsterne ist der PD oder, wenn die Sterne in der PD fehiten, den Angaben von Fost (A. N. 4564 und 4574 ) entnommen.

Seit November wird die Nova auch fortwährend photographisch beobachtet; am 30. Dez. I9I2 war sie photographisch evident schwächer als $h$. bl $=$ bloßes Auge, $\mathrm{T}=$ Trieder-Binokel, $\mathrm{S}=$ Sucher.

\begin{tabular}{|c|c|c|c|c|c|c|}
\hline \multicolumn{2}{|c|}{ I 9 I 2} & \multicolumn{2}{|c|}{$\begin{array}{c}\text { M. Z. } \\
\text { Leiden }\end{array}$} & Instr. & Schätzung & Gr. \\
\hline März & 17 & & $35^{\mathrm{m}}$ & $\begin{array}{l}\mathrm{T} \\
\mathrm{bl}\end{array}$ & $\begin{array}{llll}a & 2 & N_{2} & b \\
a & N_{1} & b\end{array}$ & $5^{\mathrm{m}} \cdot 5^{8}$ \\
\hline & 18 & 7 & 30 & $\begin{array}{l}\mathrm{T} \\
\mathrm{bl}\end{array}$ & $\begin{array}{lll}a_{1} & N_{2} & b \\
a_{1} & N_{2} & b\end{array}$ & 5.49 \\
\hline & i9 & 7 & 35 & $\begin{array}{l}\mathrm{T} \\
\mathrm{bl}\end{array}$ & $\begin{array}{llll}a & 2 & N_{2} & b \\
a_{3} & N_{1} & b\end{array}$ & $5 \cdot 5^{8}$ \\
\hline & 20 & 7 & 35 & $\begin{array}{l}\mathrm{T} \\
\mathrm{bl}\end{array}$ & $\begin{array}{llll}a & 2 N 2.5 & b \\
a & 3.5 & N_{\mathrm{I}} & b\end{array}$ & $5 \cdot 54$ \\
\hline & $2 \mathrm{I}$ & 7 & 20 & $\mathrm{~T}$ & $a_{3} N$ I $b$ & 5.67 \\
\hline & 24 & 9 & 15 & & $a_{1} 3 \cdot 5 N 2 a$ & 4.88 \\
\hline & 26 & & 2 & & $a_{4} N_{\text {I }} b$ & 5.70 \\
\hline & 27 & 7 & 45 & & $b_{3} N$ I $c$ & 6.10 \\
\hline & 28 & 9 & 25 & & $b_{3} N$ I $c$ & 6.10 \\
\hline & 29 & 10 & & & $b_{3} N$ I $c$ & 6.10 \\
\hline & $3^{\circ}$ & 9 & 20 & & $a_{4} N$ I $b$ & $5.7 \circ$ \\
\hline April & I & 9 & I 5 & & $b_{3} N$ I $c$ & 6.10 \\
\hline & 2 & 7 & 50 & & $b_{3} N 2 c$ & 6.04 \\
\hline & 3 & 8 & 5 & & $b_{2} N_{3} c$ & 5.97 \\
\hline & 4 & 8 & I 5 & & $b_{2.5} N$ I $c$ & 6.08 \\
\hline & 6 & 9 & 55 & & $b_{3} N$ I $c$ & 6.10 \\
\hline & 9 & 10 & 25 & & $b 2.5 N$ I $c$ & 6.08 \\
\hline & Io & 8 & I 5 & & $N=c, c$ I $N_{4.5} e$ & 6.24 \\
\hline & & & Io & & $c_{\mathrm{I}} N_{5} e$ & 6.28 \\
\hline & I I & 8 & 5 & & ${ }^{C} 1.5 N_{5.5} e$ & 6.33 \\
\hline & 12 & 9 & 55 & & $N=c$ & 6.18 \\
\hline & 13 & 8 & 5 & & $c 2 N 6 e$ & $6 \cdot 34$ \\
\hline & r 5 & 8 & I 5 & & ${ }^{c} 5 N_{1.5 e}$ & 6.66 \\
\hline & I 6 & 8 & 45 & & $c_{\mathrm{I}} \mathrm{N}_{4} \mathrm{e}$ & 6.30 \\
\hline & 17 & 9 & 5 & & $c 2 N 3.5 e$ & 6.40 \\
\hline & I 9 & 8 & 35 & & $c 2 N_{3.5} e$ & 6.40 \\
\hline
\end{tabular}

\begin{tabular}{|c|c|c|c|c|}
\hline 1912 & $\begin{array}{l}\text { M. Z. } \\
\text { Leiden }\end{array}$ & Instr. & Schätzung & Gr. \\
\hline April $2 c$ & $9^{\mathrm{h}} 35^{\mathrm{m}}$ & $\mathrm{T}$ & ${ }^{\circ} 5 N$ I $c$ & $6^{m} \cdot 70$ \\
\hline 2 & 95 & & $c 2 N$ I $f$ & 7.08 \\
\hline 22 & 850 & & $e$ I $N$ i $f$ & 7.01 \\
\hline 23 & 855 & & $e 2 N^{T} f$ & 7.08 \\
\hline $2 \%$ & 90 & & $N=f$ & 7.22 \\
\hline Mai & 915 & & $c 2.5 N_{1.5} e$ & 6.56 \\
\hline I 3 & 930 & & $N=g$ & 7.82 \\
\hline Aug. 2 & 1515 & & $f \perp N$ I $g$ & $7 \cdot 5^{2}$ \\
\hline Sept. & 155 & & $g=N 2 h$ & 8.08 \\
\hline
\end{tabular}

Sept. 20, 2 I, Okt. 5, 6, 8, 9, 16 und Nov. 3, 28 und Dez. 3 immer $N=h$ gefunden.

Am 27. und 28. Jan. I9I3 beobachtete ich die Nova bei sehr klarer Luft am Zehnzöller mit 600 maliger Vergrößerung und bemerkte einen Begleiter etwa I 4. Größe (beinahe an der Grenze der Sichtbarkeit) in etwa $30^{\circ} \mathrm{Po}$. sitionswinkel und ca. $3^{\prime \prime}$ Distanz. Dieser Stern ist vermutlich der Stern nördlich von der Nova auf der Karte von Wolf (A. N. $45^{64}$ ).

Weiter bemerkte ich, daß während alle Sterne der Umgebung scharf waren, ich für die Nova den Fokus I. I mm vom Objektiv $a b$ zu ändern hatte. Die Änderung fand ich an beiden Abenden bei 4 I 2-, 600- und $855^{-f a c h e r}$ Vergrößerung. Der Fokus der Nova kommt dann überein mit dem Fokus für etwa $\lambda 5_{20}\left(\mathrm{E}\right.$, grün) oder $\lambda 65^{\circ}$ (C, rot), abgeleitet aus der Kurve der chromatischen Aberration des Objektivs. Die Nova ist also vermutlich ein wenig grün, weil sie sicher nicht rot ist.

In M. N. 73.72 gibt Stratton an, daß im Dezember I9 I $\lambda_{5}$ or die zweit hellste Linie im Spektrum gewesen sei, was mit meiner rohen Einstellung am Refraktor in gutem Einklang ist.

$$
\text { Leiden, Sternwarte, I9 I } 3 \text { Febr. } 2 . \quad \text { F. Voûte. }
$$

Als ich die Nova I 9 I 2 April 2 zuerst im Sucher aufsuchte, erschien dieselbe 2 Größenklassen schwächer als $\vartheta$ Geminorum $(3 \cdot 84)$. Ich machte alsdann am Refraktor folgende Größenvergleichungen nach der Argelanderschen Methode:

1912 Gr. Zt.

April $29^{\mathrm{h}} 35^{\mathrm{m}} N 0^{\mathrm{m}}$.o PD $4200 ; N 0^{\mathrm{m}} \cdot 8$ PD $4317 \quad 5^{\mathrm{m}} \cdot 92$ 10 ro 7 PD $42000^{\mathrm{m}} 6 N$; $\mathrm{PD}_{43} 170^{\mathrm{ml}} 3 N$; $N$ om.8 PD 4243

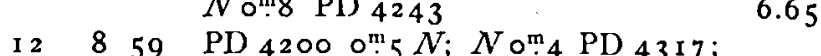

$N 0^{\mathrm{m} 8} \mathrm{PD} 4243 \quad 6.39$

2 I $95^{8} \quad \mathrm{PD}_{43}$ I 7 ०.! $N ; N 0^{\mathrm{m}} \cdot 3 \mathrm{PD}_{4243} 6.9$ I

Die Größenangaben beziehen sich auf die Skala der Potsdamer Durchmusterung.

Diisseldorf, I 9 I 3 Jan. 23.

Wilhelm Luther.

In halt zu Nr. 4634. A. N. Panoff. L'attraction universelle considérée comme fonction du temps. r 7. $-C$. Pulfich. Über Veränderungen auf dem Monde. 27. - G. F. Dodwell. Komet $1912 \mathrm{~d}$ (Lowe). 29. - Mitteilungen uber die Nova Geminorum 2. 31. 